\title{
Quality of Service Impact on Deficit Round Robin and Stochastic Fair Queuing Mechanism in Wired-cum-Wireless Network
}

\author{
Fahim Khan Khalil, Samiullah Khan \\ Farooq Faisal, Mahmood Nawaz, Farkhanda Javed \\ Institute of Business Management Sciences \\ The University of Agriculture Peshawar \\ Peshawar-Pakistan
}

Fawad Ali Khan, Rafidah MD Noor

Department of Computer System and Technology

University of Malaya, Kuala Lumpur

Malaysia

\author{
Matiullah, Zia ullah, Muhammad Shoaib, Faqir Usman Masood \\ Department of Basic Sciences and Islamiat \\ Department of Computer System Engineering \\ University of Engineering and Technology \\ Computer science Department, Qurtuba University \\ Peshawar-Pakistan
}

\begin{abstract}
The deficient round robin (DRR) and stochastic fair queue (SFQ) are the active queue mechanism (AQM) techniques. These AQM techniques play important role in buffer management in order to control the congestion in the wired-cum-wireless network by dropping packets during the buffer overflow or near to overflow. This research study focus on the performance evaluation of the DRR and SFQ using different scenarios such as increasing number of node scenario, pause time scenario and mobility scenario. We evaluate the performance of DRR and SFQ based on two parameters such as average packet delay and average packet dropped. In case of increasing number of nodes, the SFQ has outperformed than DRR by having comparatively low per packet delay. DRR has higher packet dropped ratio as compare to SFQ. In mobility and pause time scenario, SFQ has less per packet delay while DRR has less packet dropped ratio These results revealed that DRR performance was affected by an increase in the number of nodes in a network. The DRR send the packet in a round-robin fashion without caring about the bandwidth of a path due to which the packet dropped ratio was high. On another hand, the SFQ has comparatively outperformed in all scenarios by having less per packet delay. SFQ become aggressive by dropping more data packets during buffer overflow. In short, SFQ will be preferred for a network where the congestion occurred more frequently.
\end{abstract}

Keywords-Active queue management; deficit round robin; stochastic fair queuing

\section{INTRODUCTION}

To provide a wide range of connectivity to its mobile nodes wireless networks are connected with infrastructure networks. Such a scenario is known as wired-cum-wireless networks. Access Point is used as fixed base stations between wired and wireless networks [1]. In mobile ad hoc network (Manet), a number of efficient routing protocols are used, i.e. ad hoc-on demand distance vector (AODV), dynamic source routing (DSR), DRR and SFQ whose performance degraded in wired-cum-wireless scenario. In wired-cum-wireless networks, mobile host performances are affected during handoff time causes packet loss and degrade throughput [2].

Active Queue Management main function is to remove congestion, predictable queuing delay, and high link utilization, but an AQM scheme should promote high network stability, robustness, responsiveness, and scalability. To define robustness, it is important that the AQM algorithm executes constantly well under intense and unfavorable network conditions (like when changes in network parameters occur it does not perform any effect) [25]. It showed more improvement when AQM parameters tuned when there is a change in traffic load. Responsiveness defines as the speed of convergence to an equilibrium. Stability means that AQM algorithm performs static whenever there is a change in network condition. Scalability is important in AQM as it performs its functions firmly and steadily when speed and number of routers increases and number of links also increase [23].

This research focus on performance evolution of DRR and SFQ routing protocols in wired-cum-wireless scenario. DRR used different sizes of packets without caring their mean size. The packet left from one round due to its large size will be prioritized for transmission in the second round. SFQ used hashing technique in packet selection for transmission. This technique was able to map packets to the corresponding queue. SFQ reserved one queue for each flow with condition that queues number should be less than flows count [26].

The rest of the paper is organized as follows: Section II summarizes some existing AQM techniques. Section III introduced DRR and SFQ mechanism. Simulation configuration, performance analysis parameters and results analysis are mentioned in Section IV. Finally, the conclusion with future recommendation are mentioned in Section V. 


\section{RELATED WORK}

In wired-cum-wireless network, the intermediate nodes receive and forward the packets to the destination. These nodes store extra packets in the internal memory of intermediate nodes called buffer. When incoming traffic rate is larger than outgoing traffic the buffer space becomes full which results in buffer overflow by dropping data packets. This degrades the quality of service (QoS) in wired-cum-wireless scenario by increasing the delay and packet drop rate. The research problem is to find out the effect of buffer overflow on AQM techniques in wired-cum-wireless network in order to cope with abnormal delay and packet drop rate [27].

AQM techniques are used to overcome the congestion in the network by managing the packets in the buffer. In order to reduce the congestion in the network, the AQM uses intelligent packet drop system when the buffer is full or near to full. The decisions of packet drop are taken by various algorithms such as DRR and SFQ. The first AQM scheme i.e. Random Early Detection (RED) was introduced in 1993.These schemes were known as active due to its vigorously signal congestion to sources, explicitly by making packets or implicitly by dropping packets, while Drop-Tail queue is known as passive due to its dropping nature of packets when the queue is full. In 1998, the Internet Engineering Task Force (IETF) commended the deployment of AQM in internet routers. Their main task was to improve the performance and the prevention of congestion collapse which may arise from the growth of non-responsive traffic on the internet [3].

Shreedhar and Varghese (1996) found that in fair queuing process each flow was passing through a device which shared network resources [24]. Adopting such kind of network is not possible due to high expenses. DRR was a new approach in fair queuing. This scheme achieved nearly perfect fairness in terms of throughput, requires only $O(l)$ work to process a packet and was simple enough to implement in hardware. DRR could also be implemented in those scheduling problems where servicing cannot be broken up into smaller units and to distributed queues.

Kortebi and Roberts (2005) studied the performance of DRR and priority deficit round robin PDRR [15]. PDRR is an extension to DRR and used in highly dynamic networks. PDRR is more scalable than DRR. The number of flows to be looped is less than hundred at any kind of link speed. Its latency period is also very small for streaming packets. PDRR required few additional instructions to be implemented as compare to DRR [28].

Rind et al. (2006) studied that IEEE 802.11 network had various encounters like connectivity and performance problems [2]. Different routing protocols were used i.e. DestinationSequenced Distance Vector (DSDV). TCP and UDP performances were checked in wired cum wireless LAN using DSDV protocol. TCP give accurate results when numbers of moving nodes are less. File transfer rate, buffering of video and audio produced encouraging results while it is down in case of voice over IP. While UDP show better results in the case of voice over IP [19].

McKenney (2009) introduce fairness queuing method to separate dense network users from overloading prompted users [20]. According to fairness queuing method each conversation is mapped to its particular queue. There are other methods which implemented other mapping techniques but they are slow and lot of memory required. To mitigate these issues SFQ introduced. In this algorithm no exact mapping is required and also suitable for firmware implementation [29].

Cooper and Meghanathan (2010) had tried to investigate the effects of different mobility models on non-disjoint and link-disjoint multipath routing algorithms for MANET [9]. Gauss-Markov model produced least number of multi-paths and maximize lifetime per multi path. Random Direction mobility model proved the smallest lifetime per multi-path routing.

Liu et al. (2010) studied that a fair scheduling mechanism had an excellent ability and having low complexity [17]. In a communication network, it was observed that self-similar traffic was persistently presented. An analytical model was found to best for judgment of packet size effect on performance. The developed model was considered to best for analysis individual traffic flows.

Lin and Hamdi (2010) studied Fair-queuing algorithms which rely greatly on multiple queuing structures or suffered from the high time complexity which is difficult to implement in large scale due to the access delay of DRAM [16]. FQ algorithm has suffered from at least one of the difficulties in 802.16 networks, i.e. high time complexity, flow aggregation and lack of scalability. To face these challenges, they proposed a two-stage FQ algorithm, namely BRR. Their scheme worked in two steps. Furthermore they discussed the process of enqueuing and dequeuing separately [30].

Maan and Mazhar (2011) try to prove the difference between MANET and other wireless and wired networks [18]. The main difference which distinguishes between them was mobility. Here, the author compare performance of three mobility models i.e. RWP, reference point group mobility and column mobility model in MANET.Noon et al., (2011) considered the round robin (RR) algorithm to be more widely used adopted algorithm and discussed its flaws [21]. Choosing the optimal time quantum is a bottleneck in a RR algorithm. The processing time of CPU is too high for the time quantum. So selecting the proper quantum time is a major issue to solve the processing time. To overcome this problem a new approach called AN algorithm is designed which was based on dynamic time quantum instead of a fixed time quantum. Instead of the user, the operating system itself chooses the time quantum for itself. It solves the time quantum problem and improves operating system performance and increases the run time of $R R$.

Jonit and Baba (2011) had mentioned more analysis on schedule in order to have better output on scheduler performance [13]. Two different types of scheduler i.e.FIFO and DDR were compared to check their performance in 802.16. Both schedulers give same throughput for all parameters except for variable packet size. FIFO scheduler has less delay as compare to DDR. Both schedulers can be chosen based on the need of the Internet provider.

Patel et al. (2012), investigated different congestion control scheduling algorithm i.e. RED, SFQ and random exponential marking (REM) [22]. These algorithm are tested 
for the delay, throughput, and queue length parameters. Red is used to increase traffic in the network. SFQ is used for the prevention of busty flow and provide fair access to the network and REM was used for congestion measurement. Red shows better results in terms of delay. REM was best in throughput and loss ratio. S FQ shows an average in loss ratio. Among all the three algorithms REM is consider as best algorithm for a congested network.

Garg et al. (2013) analyzed the importance of management schemes for Internet and MANET [10]. Two hybrid routing protocols i.e. Zone Routing Protocol (ZRP) and LANMAR. The comparison of the system proved that the LANMAR system is more feasible and beneficial for the use. Veni and Latha (2013) studied that each device and equipment in MANET model move freely and independently in any direction. In such model, the main challenge is continuously maintaining the required and proper information. MANET has the capability to change the location and adapt itself according to requirement. In such case, the network is decentralized where all network activities are incorporated in mobile path.

According to Gupta et al. (2013) MANET system contained a variety of mobile node which can change topology easily. It did not require centralized infrastructure [?]. Three different routing protocols i.e. AODV, DSR, and DSDV were used in various mobility models. If mobility rate is high DSDV performance will be low. The better delivery ratio of DSR and AODV depends on discovery of a route through which data can be transmitted to the ultimate destination. These routing protocols adopt different mechanism in case of frequent link failure due to mobility.

Goyal and Kakar (2013) studied that various mobile nodes collectively form an ad hoc network and communication with a wireless link [11]. MANET was connected with wireless transmitter and receivers. The author compared the performance of four reactive routing protocols i.e. Dynamic MANET on Demand (DYMO), location aided routing (LAR) AODV, DSR. LAR protocol outperformed by having less jitter and end to end delay. Alsahag et al. (2014) studied the rapid advancement of technologies in computer networking and required QOS to manage its overall performance [6]. A Worldwide Interoperability for Microwave Access (WIMAX) network has been studied and a special bandwidth allocation technique has been applied. One of the major issue in WiMAX is its scheduling algorithm. In real-time and non-real-time application WiMAX did not fulfill the requirements of QOS which lead to insufficient allocation of bandwidth, latency, and throughput. To overcome these issues the author proposed a new scheme called FADRR for mobile WiMAX. For real and non-real time application FADRR uses fuzzy logic to allocate bandwidth that guarantees the optimal bandwidth for each flow.

Alsahag et al. (2014) studied the rapid advancement of technologies in computer networking and requiring quality of service QOS to manage its overall performance [6]. A WIMAX network has been studied and a special bandwidth allocation technique has been applied. One of the major issues in the WiMAX was its scheduling algorithm is real-time and nonreal-time application that did not fulfill the requirement of the quality of services which lead to insufficient allocation of bandwidth, latency, and throughput. This paper introduces a new scheme called FADRR is propped for mobile WIMAX. FADRR used fuzzy logic approach and different service flows in BS. FADRR used a deadline based approach to allocating bandwidth for real and non-real time application and this bandwidth allocation is done by mean of a fuzzy logic system that guarantees the optimal bandwidth for each flow by taking latency and throughput parameters under its consideration. FADRR has also been evaluated for a number of different algorithms like MDRR and CDRR by taking jitter, delay, throughput and fairness under its consideration for different classes like arts, reps, nrtps, ugs and be but FADRR proved to be the best among all. The simulation results analyzed that FADRR was efficient in real time applications in respect to QOS while it's a lot fair allocation to non-real time application and improves the overall system performance.

Chitkara and Ahmad (2014), studied that laptops, wireless telephones, and wireless sensors were mostly used nowadays [7]. A wireless node having no infrastructure and no central administration were used in MANET. Topology was also changed very frequently between the nodes. Different routing techniques and different strategies were implemented in MANET. In MANET, the authors have studied different characteristics, advantages, application and challenges in Manets.

In short, there were many AQM techniques proposed in the literature that was specially designed for wired network or wireless networks such as RED, Droptail [5] Blue [22] and ECN [8]. These AQM techniques had their own pros and cons in various scenarios as discussed above. In literature survey, there were no specialized techniques that were well designed for wired cum wireless environment. On another hand, the wired cum wireless networks popularity increased day by day due to technological advancements such as laptops, smartphones, and tablets. This literature study revealed the importance of DRR and SFQ utilization in wired cum wireless network which can help us in formulating wired cum wireless base specialized AQM technique.

\section{ACtive QueUe MANAGEMENT}

The main function of AQM is to remove congestion, predictable queuing delay, and high link utilization. There are three queue management schemes used in AQM components. namely congestion indicator, congestion control function, and feedback mechanism [4]. When there is congestion in the network the queue management uses congestion indication to decide when there is congestion. Whereas how to remove the congestion or what must be done when there is congestion, it's the duty of congestion control function. The function of the feedback mechanism is the congestion signal used to aware the source to adjust its transmission rates [23]. These components are shown in Fig. 1.

DRR and SFQ are AQM techniques play important role in buffer management in order to control the congestion in the wired-cum-wireless network by dropping packets during the buffer overflow or near to overflow [14].

\section{A. Deficit Round Robin}

Simple round robin service uses the constant time for packet scheduling along different paths. The major flaw in simple round robin scheduler is to avoid large packet size in the 


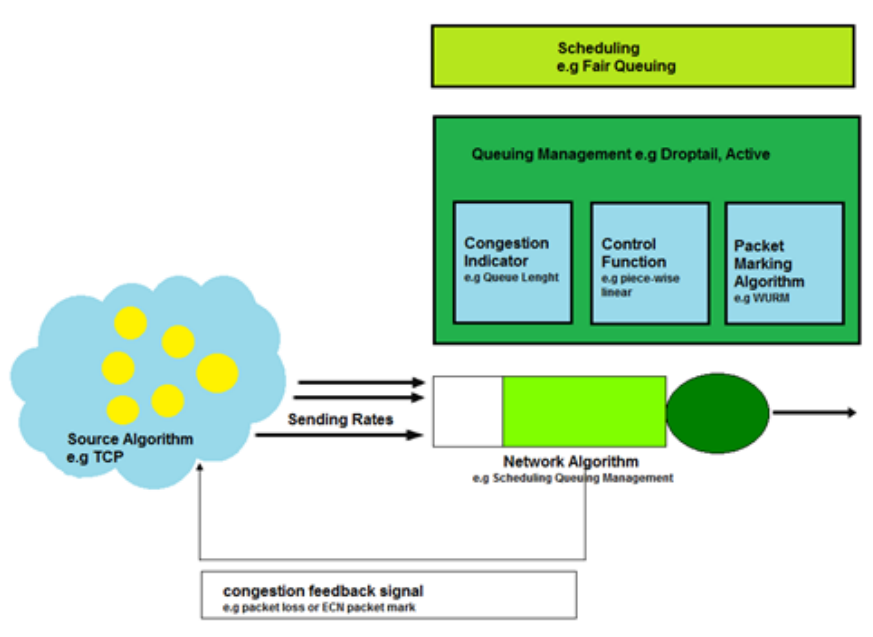

Fig. 1. General active queue management components.

first round. Variation in packet size creates unfairness which can be removed when the time is constant. This modification of round robin service is called DRR [24].

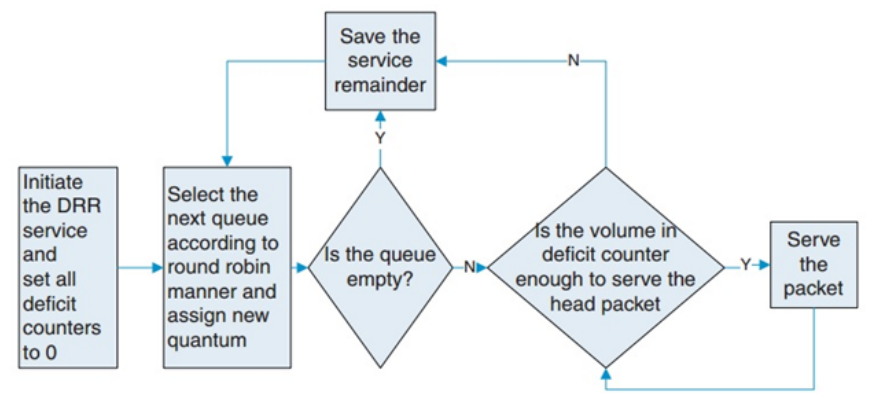

Fig. 2. Flow diagram of DRR mechanism.

DRR scheduling transmits traffic flows in a round robin manner. Unlike other round robin variants, DRR provides deficit counters for individual traffic flows. A volume of service equal to its quantum size is provided to each active flow in every round. The remaining volumes of services are used in the next round for deficit counter. This means at the start of each round the volume of the deficit counter of a traffic flow is equal to the sum of its quantum size and the volume of its remaining service from the previous round. An active flow has the packets that can be served in each round are determined by the value of its deficit counter. The DRR scheduling mechanism initiates to attend the next active flow in each round under either of the two conditions. The present queue is either blank or there is not enough service volume left to serve packet in the current flow. There is a static quantum given to each flow to avoid misbehavior in traffic flow. The DRR can also eliminate the unfairness, caused in the changeability of packet sizes of different flows. This is truly the most important improvement of DRR compared to the original round robin scheme. It is clear that the service assigned to each flow depends only on its fixed quantum and any greedy traffic flows cannot take away the remaining service of the other flow [17]. Flow diagram of DRR is shown in Fig. 2.

\section{B. Stochastic Fair Queue}

Scheduling algorithm uses fair queuing technique. Fair queuing is used in a network scheduler. In every traffic flow, a separate data packet queue is used contrasting to the FIFO queue technique which uses a single queue for all data packet flows. Fairness is only accomplished when a small amount of resources is used. The fair queuing algorithm uses SFQ technique. Stochastic fair queue performance is not precise as compared to others but it is best according to fewer calculations. Conversation (or flow) is the main word which is often used in SFQ coincides with TCP session.

\section{Stochastic Fair Queuing (SFQ)}

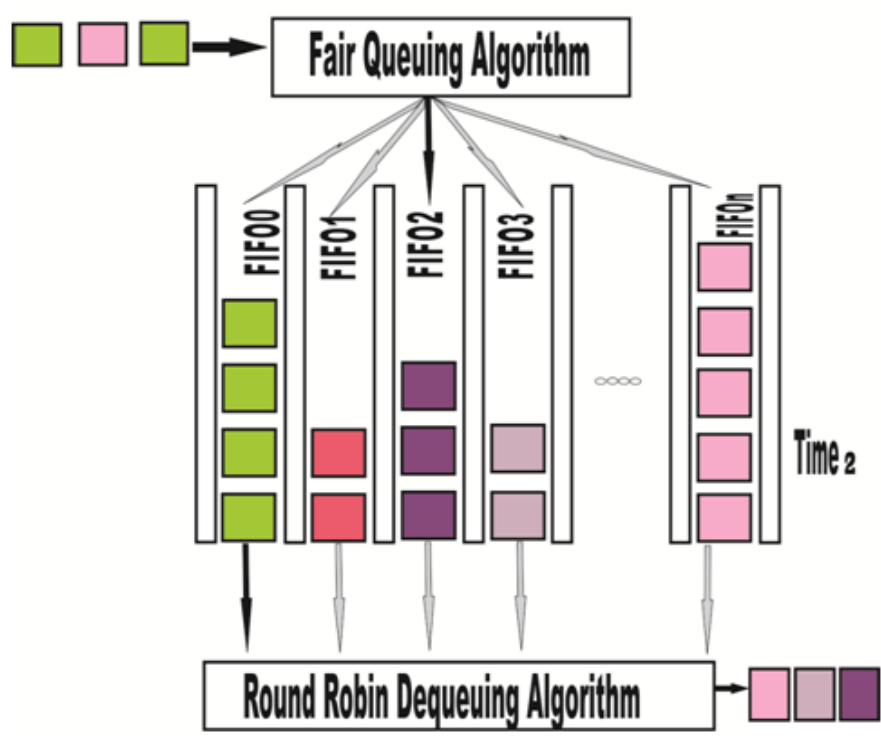

Fig. 3. Working diagram of stochastic fair queue.

When large amount of traffic is coming it is separated into FIFO queues using a single flow for each queue. Here a round robin technique is used for a huge traffic because every session has the equal chance to send their data. This is the most efficient process for every conversation and it does not drown the entire flow. SFQ uses an algorithm known as hashing algorithm, in this algorithm traffic is separated into a number of queues and does not assign queue in favor of every session. Fig. 3 shows stochastic fair queuing algorithm.

\section{Performance Evaluation}

In this section, we describe simulation scenario with related parameters. The performance of DRR and SFQ are evaluated by a series of simulations using NS-2 tool [12]. In this research, file transfer protocol (FTP) is used at the application layer and transmission control protocol (TCP) is used at the transport layer. Destination sequenced distance vector (DSDV) routing protocol is used having simulation area of $250 \times 250 \mathrm{~m} 2$ and simulation time is 300 seconds. Two numbers of wired nodes, five to fifteen mobile nodes with one base station (BS) are used. Random Way Point (RWP) is used as a mobility model. There is variation in time regarding mobility. DRR and SFQ are used as an AQM technique and the buffer capacity is 50 packets. The other parameters are listed in Table I. 
TABLE I. SCENARIO CONFIGURATION PARAMETERS IN NS-2

\begin{tabular}{|l|l|l|}
\hline $\begin{array}{l}\text { Sr. } \\
\text { No. }\end{array}$ & Parameters & Values \\
\hline 1 & Application layer Protocol (ALP) & File Transfer Protocol (FTP) \\
\hline 2 & Transport Layer Protocol & $\begin{array}{l}\text { Transmission Control Protocol } \\
(\mathrm{TCP})\end{array}$ \\
\hline 3 & Number of Base Station & 1 (Basestation) \\
\hline 4 & Number of Wired Node & 2 (Wired Nodes) \\
\hline 5 & Number of Mobile Nodes & $5,10,15$ (Mobile Nodes) \\
\hline 6 & Mobility Model & Random Way Point (RWP) \\
\hline 7 & Mobility Speed & $1.4,3.3,11.11$ (m/Sec) \\
\hline 8 & $\begin{array}{l}\text { Active Queue Management Tech- } \\
\text { niques }\end{array}$ & $\begin{array}{l}\text { Deficit Round Robin (DRR) } \\
\text { Stochastic Fair Queue (SFQ) }\end{array}$ \\
\hline 9 & General Buffer Capacity in nodes & 50 Packets \\
\hline 10 & $\begin{array}{l}\text { Routing Protocol } \\
\text { Destination Sequence Distance } \\
\text { Vector (DSDV) }\end{array}$ \\
\hline 11 & Simulation Area & 250 x 250 (m2) \\
\hline 12 & Simulation Time & 300 Seconds \\
\hline 13 & $\begin{array}{l}\text { Bandwidth between Wired Node } \\
\text { / Base Station }\end{array}$ & 5 Mbps \\
\hline 14 & $\begin{array}{l}\text { Delay between } \\
\text { Nodes/Base Station }\end{array}$ & 2 ms Seconds \\
\hline
\end{tabular}

\section{A. Simulation Parameters}

In this section, we introduce some metrics that are necessary for performance evaluation. The parameter used for the QoS in the wired-cum-wireless network is average per packet delay and average packet dropped.

1) Average per packet delay: This parameter is used to find out the time consumed by the packet from one node to another node. The unit used in the end-to-end delay is a millisecond. Mathematical formula is as under.

$$
\text { Averageperpacketdelay }=\frac{\sum_{i=1}^{n} \text { EndtoEndDelay }}{\text { Numberof Packets }}
$$

2) Average packet drop rate: A number of packets dropped at a specific time is to find out by using packet dropped parameter. The unit for the packet dropped is packets per second. Mathematical formula for the packet dropped is as under:

$$
\text { conAveragepacketdropped }=\frac{\sum_{i=1}^{n} \text { DroppedPackets }}{\text { SimulationTime }}
$$

\section{B. Simulation Scenarios}

In this research study a simulation scenario is configured. On the basis of DRR and SQF different scenarios, i.e. increasing number of nodes, mobility and pause time are compared and analyzed to find out performance parameters, i.e. average per packet delay and average packet dropped.

\section{Increasing Number of Nodes Scenario}

DRR and SQF are the two AQM techniques which are observed by increasing the number of nodes from 5 to 15 while mobility and pause time remain constant.

1) Average per packet delay: The average packet delay is compared with DRR and SFQ, as shown in Fig. 4. Here it is clearly mentioned that DRR has the highest delay while SFQ has the lowest delay. As the number of nodes increases, packet delay in DRR also increases, on the other hand, using the same amount of nodes, SFQ gives the lowest delay. In short, SFQ outperformed DRR.

Using the scenario of DRR, when the numbers of nodes are less i.e. 5 nodes, the average per packet delay is less but as the number of nodes increases i.e. 15 nodes the average per packet delay also increases. It is because packets are organized in round robin. Using the scenario of SFQ, when the numbers of nodes are less i.e. 5 nodes, the average per packet delay is less but when the number of nodes increases i.e. 15 nodes, the average per packet delay is not that high as compared to DRR. It is because probability distribution and queues maintained statistically.

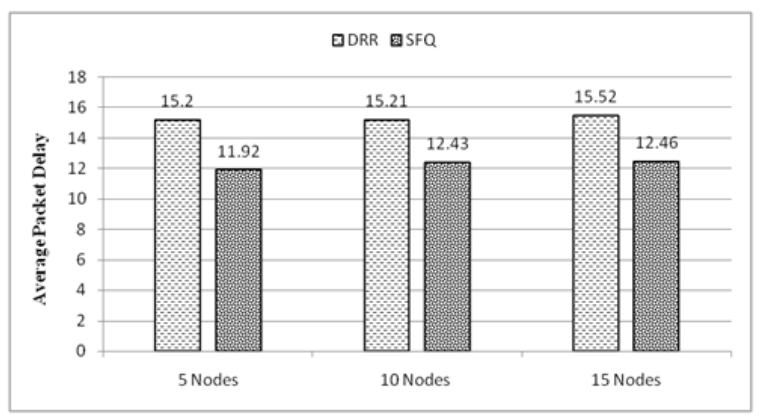

Fig. 4. Average per packet delay with respect to increasing number of nodes.

2) Average packet drop rate: Fig. 5 shows the average packet dropped between DRR and SFQ. Here, DRR has higher packet drop rate as the number of nodes are increasing from 5 to 15 . When the number of nodes is 5 less packet are dropped in DRR, as the number of nodes increases the packet dropped ratio also increases. While on the other hand SFQ shows a slight difference in packet dropped rate. When the number of nodes is less i.e. 5 nodes, packet dropped ratio is high but as the number of nodes increases i.e. 15 nodes, the dropped ratio becomes low. In short, SFQ outperforms DRR with respect to packet dropped rate.

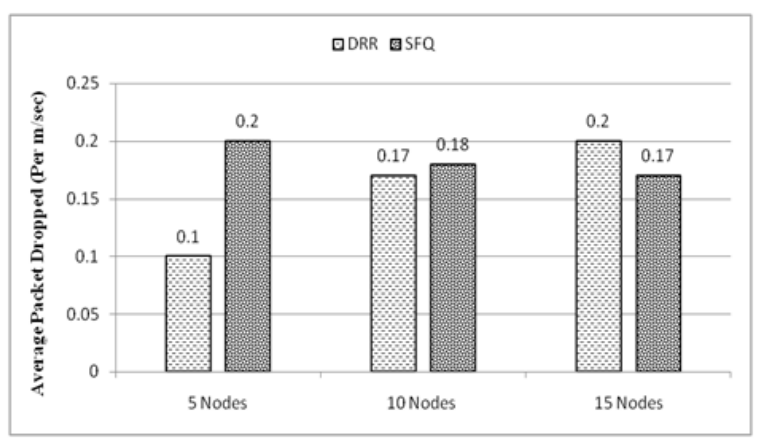

Fig. 5. Average packet drop with respect to increasing number of nodes.

\section{Mobility Scenario}

Two AQM techniques i.e. DRR and SFQ are used to compare mobility of nodes using different moving scenarios. Walking speed is $1.4 \mathrm{~m} / \mathrm{sec}$, running speed is $3.3 \mathrm{~m} / \mathrm{sec}$, and the speed of the vehicle is $11.11 \mathrm{~m} / \mathrm{sec}$. During mobility of nodes, other parameters like Pause time and number of nodes are kept constant. 
1) Average per packet delay: Average packet delay of the AQM techniques i.e. DRR and SFQ are compared using multiple moving scenarios as shown in figure 6 . It is clearly observed that walking speed, running speed and vehicle speed is kept changing like when a node is on walking speed delay is 15.21 , when a node is running, the delay is lower i.e. 15.18 , but when a node is in the vehicle delay is again rise to 15.23, the delay is almost same. This is in the case of sub-scenario of DRR, while in the case of SFQ packet delay in gradually increasing from walking speed to vehicle speed due to movement of nodes and links. Delay increases in SFQ because when nodes move distance between the nodes increasing and found problems in connectivity.

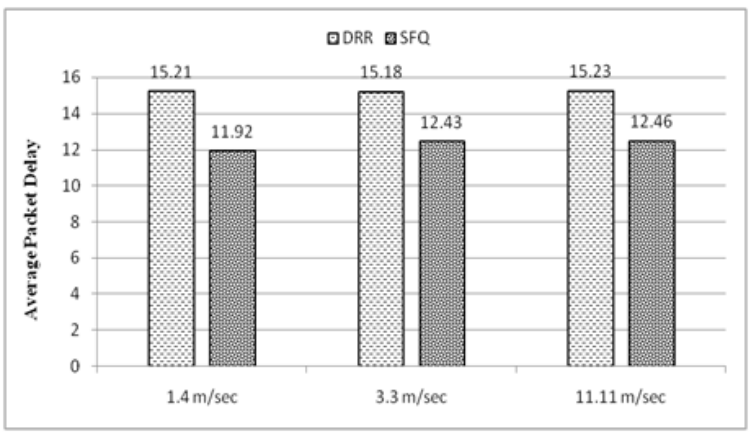

Fig. 6. Average per packet delay with respect to increasing mobility speed.

2) Average packet drop rate: Average packet dropped off the AQM techniques i.e. DRR and SFQ are compared using multiple moving sub-scenarios as shown in Fig. 7. It is clearly observed that average packet dropped rate is lower in walking speed $(1.4 \mathrm{~m} / \mathrm{sec})$ for DRR while it is higher in SFQ. When the node is in running $(3.3 \mathrm{~m} / \mathrm{sec})$ position average packet dropped rate is lower for DRR and higher for SFQ. Average packet dropped rate is lower in vehicle speed $(11.11 \mathrm{~m} / \mathrm{sec})$ for DRR while it is higher in SFQ. Average packet dropped rate is gradually increased when mobility of nodes increases for DRR.

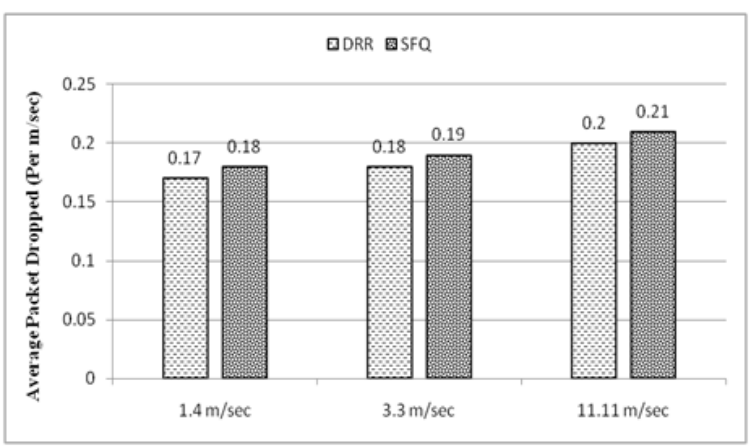

Fig. 7. Average packet dropped with respect to increasing mobility speed.

\section{E. Pause Time Scenario}

In this scenario number of nodes and mobility remain constant while pause time changes for DRR and SFQ.

1) Average per packet delay: Average packet delay is compared to AQM techniques i.e. DRR and SFQ in Fig. 8. It is observed that in each sub scenario pause time varies (between 2 to 10 seconds) for AQM techniques. In each sub-scenario, average packet delay is lowered both for DRR and SFQ.

When the pause time is 2 seconds, average per packet delay is highest for DRR and lowest for SFQ. When the pause time is 5 seconds, average per packet delay is highest for DRR and lowest for SFQ. When the pause time is 10 seconds, average per packet delay is highest for DRR and lowest for SFQ. It is observed from Fig. 4 and 5, as the time increases the node become too static, so the delay in the packet is lowered due to network link failure and stable network topology.

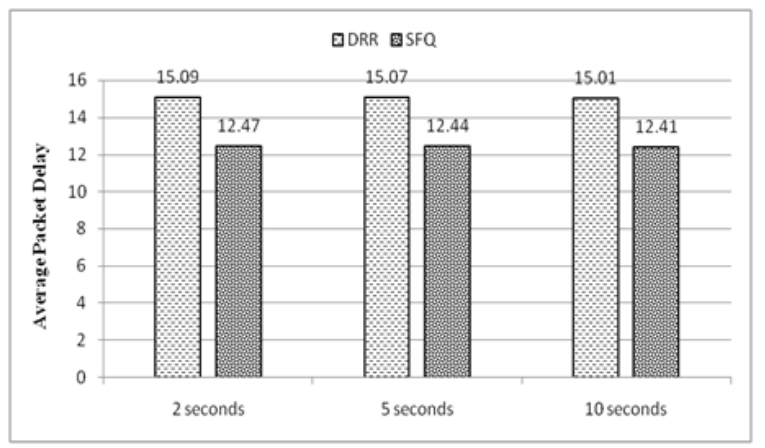

Fig. 8. Average per packet delay with respect to increasing pause time.

2) Average Packet Drop Rate: Average packet drop rate is gradually decreasing for $\mathrm{AQM}$ techniques i.e. DRR and SFQ by changing pause time. It is observed from Fig. 9 that in each sub scenario i.e. 2, 5 and 10 seconds pause time varies. Mostly average packet drop rate for DRR and SFQ is lowered due to low movement of nodes and no changes of intermediate nodes between sender and receiver. It uses the same route as established at the time of connection, no need to find a new route. As time increases the node come to static state, so no disconnection occurs.

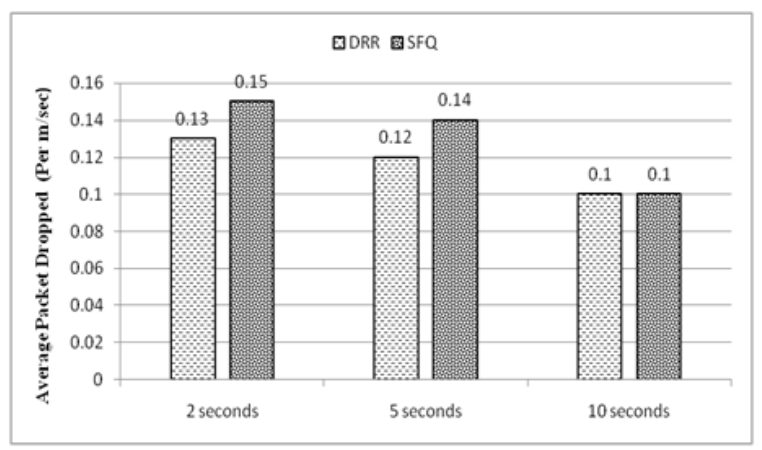

Fig. 9. Average packet drop with respect to increasing pause time.

\section{CONCLUSiOn}

This research paper focus on the performance evaluation of the DRR and SFQ using different scenarios such as increasing number of nodes, pause time and mobility scenario based on two parameters. It is observed the SFQ has outperformed than DRR by having comparatively low per packet delay. While on the other hand DRR has highest packet ratio as compare to 
SFQ in case of increasing number of nodes. In mobility and pause time scenario, SFQ has less per packet delay while DRR has less packet dropped ratio. Considering all the evaluation and results, it is necessary to analyze AQM techniques with different traffic pattern in wired cum wireless network such as bursty traffic and constant bit rate. Furthermore, it is also necessary to modify SFQ to drop data equally from all flows instead of targeting single flow. DRR should be implemented with limited number of queues to improve its performance for future work.

\section{REFERENCES}

[1] Wang, X., Kar, K., and Low, S. H. (2009). End-to-end fair rate optimization in wired-cum-wireless networks. Ad Hoc Networks, 7(3), 473-485

[2] Rind, A. R., Shahzad, K., and Qadir, M. A. (2006). Evaluation and comparison of TCP and UDP over Wired-cum-Wireless LAN. In Multitopic Conference, 2006. INMIC'06. IEEE (pp. 337-342).

[3] Bitorika, A., Robin, M., Huggard, M., and Mc Goldrick, C. (2004). A comparative study of active queue management schemes. In Proc. of ICC.v2004

[4] Adms, R. (2013). Active queue management: a survey. IEEE communications surveys and tutorials, Vol. 15, Issue 3, pp-1425-1476.

[5] Ahmed, I., Badia, L., and Hussain, K. (2010). Evaluation of deficit round robin queue discipline for real-time traffic management in an RTP/RTCP environment. InFourth UKSim European Symposium on IEEE Computer Modeling and Simulation (EMS), pp. 484-489.

[6] Alsahag, A. M., Ali, B. M., Noordin, N. K., and Mohamad, H. (2014). Fair uplink bandwidth allocation and latency guarantee for mobile WiMAX using fuzzy adaptive deficit round robin. Journal of Network and Computer Applications, Vol.39, pp.17-25.

[7] Chitkara, M., and Ahmad, M. W. (2014). Review on MANET: Characteristics, Challenges, Imperatives and Routing Protocols. International Journal of Computer Science and Mobile Computing, 3 (2), 432-437.

[8] Chung, J., Claypool, M., and Kinicki, R. (2007,). Stochastic Fair Traffic Management for Efficient and Robust IP Networking. In Performance, Computing, and Communications Conference, 2007. IPCCC 2007. IEEE International (pp. 45-54)

[9] Cooper, N., and Meghanathan, N. (2010), Impact of mobility models on multipath routing in mobile ad hoc networks, AIRCC Int. J. Comput. Netw. Commun, 2, 174-185.

[10] Garg, P., Nagpal, C. K., and Bansal, S. (2013). The impact of Random Waypoint Mobility Model on Hybrid Routing Protocols of Scalable Mobile Ad Hoc Network. International Journal of Innovative Research and Development, 2(10).

[11] Goyal, E. H., and Kakkar, E. P. (2014,). Performance Investigation of DYMO, DSR, AODV and LAR Routing Protocols using Different Mobility in MANETs. In International Journal of Engineering Research and Technology (Vol. 2, No. 12 (December-2013)). ESRSA Publications.

[12] Issariyakul, T., and Hossain, E. (2011). Introduction to network simulator NS2. Springer Science and Business Media, pp 35-36

[13] Jonit, N. M., and Baba, M. D. (2011). First in first out (FIFO) and Deficit Round Robin (DRR) scheduling analysis in WiMAX network. In Control and System Graduate Research Colloquium (ICSGRC), 2011 IEEE (pp. 166-174).
[14] Jain, B., and Madan, S. Comparative Analysis of various Active Queue Management Algorithms under Flooding-based LDDoS Attack.

[15] Kortebi, A., Oueslati, S., and Roberts, J. (2005). Implicit service differentiation using deficit round robin. ITC19.

[16] Lin, D., and Hamdi, M. (2010,). Two-stage fair queuing using budget round-robin. In Communications (ICC), 2010 IEEE International Conference on (pp. 1-5).

[17] Liu, L., Jin, X., Min, G., and Li, K. (2010). Performance modeling and analysis of Deficit Round Robin scheduling scheme with self-similar traffic. Concurrency and Computation: Practice and Experience, 22(13), 1911-1926.

[18] Maan, F., and Mazhar, N. (2011). MANET routing protocols vs. mobility models: A performance evaluation. In Ubiquitous and Future Networks (ICUFN), 2011 Third International Conference on (pp. 179-184).

[19] Mahdipour, E., Rahmani, A. M., and Aminian, E. (2009). Performance evaluation of destination-sequenced distance vector (DSDV) routing protocol. In Future Networks, 2009 International Conference on (pp. 186-190).

[20] McKenney, P. E. (1990). Stochastic Fairness is queuing. In IEEE INFOCOM'90, Ninth Annual Joint Conference of the IEEE Computer and Communication Societies. The Multiple Facets of Integration. Proceedings, IEEE (pp. 733-740).

[21] Noon, A., Kalakech, A., and Kadry, S. (2011). A new round-robin based scheduling algorithm for operating systems: dynamic quantum using the mean average. arXiv preprint arXiv:1111.5348.

[22] Patel, S., Gupta, P. K., Garg, A., Mehrotra, P., and Chhabra, M. (2012). Comparative analysis of congestion controls algorithms using ns-2. arXiv preprint arXiv: 1203.3654

[23] Radhakrishnan, S., Pan, R., Vahdat, A., and Varghese, G. (2012). Netshare and stochastic net share: predictable bandwidth allocation for data centers. ACM SIGCOMM Computer Communication Review, 42(3), 5-11.

[24] Shreedhar, M., and Varghese, G. (1996). Efficient fair queuing using deficit round robin. Networking, IEEE/ACM Transactions on, 4(3), 375385.

[25] Khan, S. and Qadir, M. A. (2015). Inter-path OOS packets differentiation based congestion control for simultaneous multipath transmission. Int. Arab J. Inf. Technol. Vol.4, No.6, pp.907-913.

[26] Khan, S. and Qadir, M. A. (2017). Deterministic Time Markov Chain Modelling of Simultaneous Multipath Transmission Schemes. IEEE Access, Vol. 5, pp.8536-8544.

[27] Ali, H., Khan, S. and Quaid, M. (2015). Comparative analysis of controlled delay (CoDel) with Deficit Round Robin (DRR) to overcome bufferbloat problem in wired network. International Journal of Current Engineering and Technology, Vol.5, No. 5, pp. 3378-3386.

[28] Khan, F., Abbas, S. and Khan, S. (2016). An Efficient and Reliable Core-Assisted Multicast Routing Protocol in Mobile Ad-Hoc Network. International journal of advanced computer science and applications, Vol. 7, No. 5, pp. 231-242.

[29] Shakir, S. Khan,S. Hassain, L. and Matiullah (2017) QoS Based Evaluation of Multipath Routing Protocols in Manets, Advances in Networks. Vol. 5, No. 2, 2017, pp. 47-53. doi: 10.11648/j.net.20170502.13

[30] Khan, F., Abbas, S. and Khan, S. (2018). Secure Core-Assisted Multicast Routing Protocol in Mobile Ad-Hoc Network, Journal of Internet Technology, Article In Press. 